\title{
Nowcasting the Output Power of PV Systems
}

\author{
Marius Paulescu ${ }^{1, *}$, Oana Mares $^{2}$, Ciprian Dughir ${ }^{2}$, and Eugenia Paulescu ${ }^{1}$ \\ ${ }^{1}$ Faculty of Physics, West University of Timisoara, V Parvan 4, 300223 Timisoara, Romania \\ ${ }^{2}$ Department of Physical Foundations of Engineering, Politehnica University Timisoara, V Parvan 2, \\ 300223 Timisoara, Romania \\ 33Electronics and Telecommunications Faculty, Politehnica University Timisoara, V Parvan 2, \\ 300223 Timisoara, Romania
}

\begin{abstract}
This paper presents an innovative procedure for nowcasting the energy production of PV systems. The procedure is relayed on a new version of two-state model for forecasting solar irradiance at ground level and a simplified description of the PV system. The results of testing the proposed procedure against on field measured data are discussed. Generally, the proposed procedure demonstrates a better performance than the main competitor based on ARIMA forecasting of the clearness index.
\end{abstract}

\section{Introduction}

Unlike the power generated by the traditional power plants based on fossil or nuclear fuels, the output power of the photovoltaic (PV) plants is highly variable to erratic. This is due to passing clouds which may cause a fast variation of the solar irradiance inducing further a massive shift in the output power of a PV plant [1]. In this context, forecasting the power generated by PV plants becomes a critical task for smart management of the grid. Accurate forecasts will enable the computers to take control actions aiming to balance the power grid in real-time. Since there are circumstances when the fluctuations of the solar irradiance are on a time scale of minutes or less, nowcasting the output power of PV plants becomes a timely research area [1-3].

This paper presents a new procedure for nowcasting the output power of a PV plant. The procedure involves two interrelated issues: (1) forecasting the solar resource availability and (2) realistic modelling of the PV converter response. The basic concept of the proposed procedure is based on several innovative results obtained by our team: (1) a new procedure of solving the five-parameter model of a solar cell [4], (2) accurate modelling of the PV module operation in real environmental conditions [5] and (3) the innovative two-state model for short-term forecasting solar irradiance [6] based on sunshine number $(S S N)$, a binary quantity stating whether the Sun is shining or not.

The paper is organized as follows. Section 2 is devoted to description of the relevant data. The proposed procedure is introduced in Sec. 3. Since the accuracy of nowcasting the output power of a PV system is conditioned by the accuracy of nowcasting the solar irradiance, Sec. 3. is mainly focused on this issue. The results of testing the proposed procedure against measured data are discussed in Sec.4.

\footnotetext{
* Corresponding author: marius.paulescu@e-uvt.ro
} 

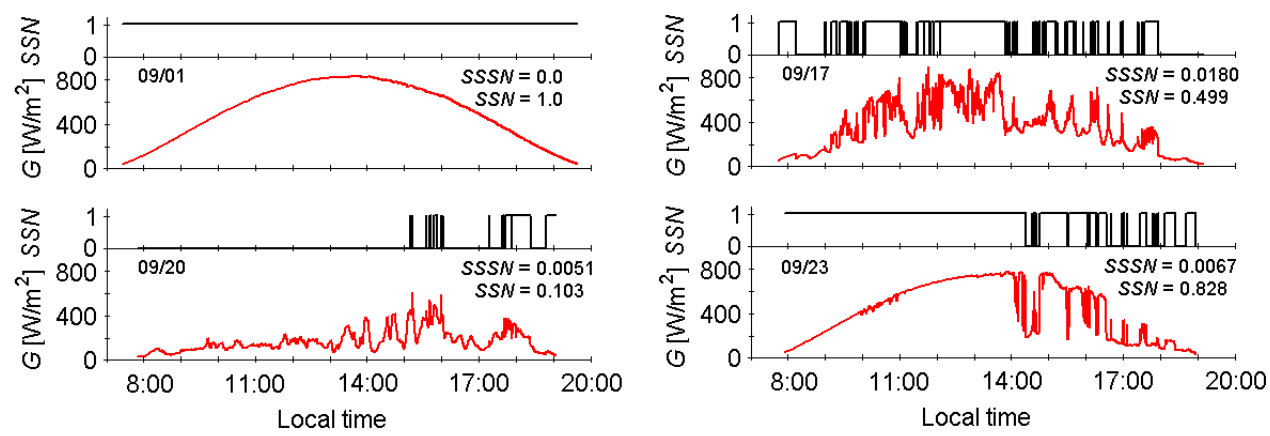

Fig. 1. Variation in time of the global solar irradiance $(G)$ and sunshine number $(S S N)$ recorded in four different days of 2016. The average values of $S S N$ and $S S S N$ are displayed on each graph.

\section{Data}

The study was conducted with data recorded on the Solar Platform of the West University of Timisoara, Romania (http://solar.physics.uvt.ro/srms). The town of Timisoara $\left(45^{\circ} 46^{\prime} \mathrm{N} ; 21^{\circ} 25^{\prime} \mathrm{E}\right.$ and $85 \mathrm{~m}$ asl) has a warm temperate climate, typical for the Pannonia Basin (Köppen climate classification $C f b$ ). The built database contains radiometric, meteorological and electrical data collected from an experimental PV setup. All the sensors are integrated into an acquisition data system based on National Instruments PXI Platform and explored simultaneously at high temporal resolution (15 seconds). The global and diffuse solar irradiance are measured using DeltaOHM LP PYRA 02 first class pyranometers which fully comply with ISO 9060 standard and meet the requirements defined by the World Meteorological Organization (WMO).

\subsection{Post-processed data}

Mathematically, the sunshine number is defined as a time dependent random binary variable, as follows [7]:

$$
S S N_{t}= \begin{cases}0 & \text { if the sun is covered by clouds at time } t \\ 1 & \text { otherwise }\end{cases}
$$

Series of $S S N$ values have been derived using the WMO sunshine criterion [8]: the Sun is shining at time $t$ if the direct solar irradiance exceeds $120 \mathrm{~W} / \mathrm{m}^{2}$. Therefore:

$$
S S N_{t}= \begin{cases}1 & \mathrm{IF}\left(G_{t}-G_{d, t}\right) / \sin h_{t}>120 \mathrm{~W} / \mathrm{m}^{2} \\ 0 & \text { otherwise }\end{cases}
$$

where $G_{t}$ and $G_{d, t}$ denote the measured global and diffuse solar irradiance at the time $t$ and $h_{t}$ is the Sun elevation angle. On basis of $S S N$ a straightforward quantifier for the variability of the solar radiative regime is defined in Ref. [9]. This is the sunshine stability number:

$$
S S S N_{t} \equiv\left\{\begin{array}{l}
1 \text { if } S S N_{t}>S S N_{t-1} \\
0 \text { otherwise }
\end{array}\right.
$$

The average value of $S S S N$ during a time interval $\Delta t, \overline{S S S N}$, ranges between 0 and $1 / 2$, measuring the frequency of $S S N$ the changes during $\Delta t$. Daily values of $\overline{S S S N}$ have been 
calculated in this study. Figure 1 illustrates the time series of $G$ and $S S N$ in four days of 2016. In every day, more than 2500 records are used to build the graph.

Another post-processed quantity is clearness index [10]:

$$
k t_{t}=G_{t} / G_{e, t}
$$

where $G_{t}$ and $G_{e, t}$ are the global solar irradiance measured at ground level and its counterpart estimated at the top of the atmosphere. Clearness index isolates the stochastic components in solar irradiance time series. The traditional approach in nowcasting solar irradiance is based on the statistical modeling of the clearness index time series.

\section{Model}

The proposed procedure for nowcasting the output power of a PV system runs two algorithms: (1) nowcasting the solar irradiance and (2) modelling the PV system. Both algorithms are briefly described next.

\subsection{The 2-state model v.2}

In this study an upgraded version of the two-state model for nowcasting solar irradiance [6] is tested. The new version of the model connects an empirical model for estimating the clear sky solar irradiance with a statistical model for forecasting $S S N$ :

$$
\hat{G}_{t}=\left\{\begin{array}{lll}
c_{c S} \cdot G_{0, t} & \text { if } & S \hat{S} N_{t}=1 \\
\bar{\tau}_{c} \cdot G_{0, t} & \text { if } & S \hat{S} N_{t}=0
\end{array}\right.
$$

where $\hat{G}_{t}$ is the forecasted value of the solar irradiance at time $t . G_{0, t}$ is the estimated solar irradiance under clear sky at time $t c_{c s}$ is a dynamic correction applied to the mean atmospheric transmittance encapsulated into the $G_{0, t}$ equation. $\bar{\tau}_{c}$ is the attenuation applied to $G_{0, t}$ according to the cloud transmittance. Note that the sole parameter that needs to be forecasted in Eq. (5) is $S S N$. The model operates as follows ( $S \hat{S} N_{t}$ denotes the forecasted value of $S S N$ at time $t$ ). On the state $S \hat{S} N_{t}=1$, the global solar irradiance is being estimated using a clear-sky model. In the first version of the two-state model $c_{c s}$ was assumed equal to one. On the state $S \hat{S} N_{t}=0$, the clear-sky estimate is adjusted with the cloud transmittance $\bar{\tau}_{c}$. Both parameters $c_{c s}$ and $\bar{\tau}_{c}$ are estimated simultaneously by a linear regression applied to data measured in a period $\Delta t$ prior to the forecasting moment. Regarding $\Delta t$, an important finding was reported in [11]: the minimum error is reached if $\Delta t$ equals the forecasting lead time.

\subsection{PV convertor}

The algorithm for modelling the PV converter combines two results obtained by our team. Firstly, the PV module current-voltage characteristics at STC is evaluated in terms of onediode model. The model parameters are extracted using the simple procedure from Ref. [4]. Secondly, the procedure from [5] is applied for translating the I-V curve from STC to an arbitrary operating point defined by the in-plane solar irradiance and the solar cell temperature. 

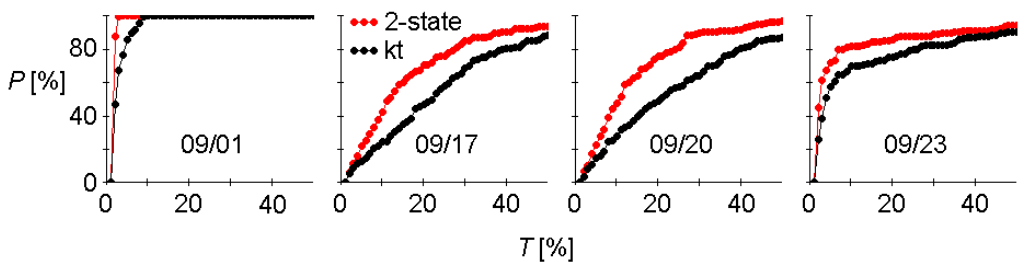

Fig. 2. Percentage of forecasts $P$ accurate to within a given tolerance interval $T$ centered on the measurements. Lead time $=5$ minutes.

Table 1 Models performance in nowcasting solar irradiance. $P(T=5 \%)$ is the percentage of forecasts accurate to within $T=5 \% . N$ represents the total number of measurements. Lead time $=5 \mathrm{~min}$.

\begin{tabular}{|c|c|c|c|c|c|c|c|c|}
\hline Date & \multicolumn{2}{|c|}{$\mathbf{0 9 / / 0 1}$} & \multicolumn{2}{c|}{$\mathbf{0 9 / 1 7}$} & \multicolumn{2}{c|}{$\mathbf{0 9 / 2 0}$} & \multicolumn{2}{c|}{$\mathbf{0 9 / 2 3}$} \\
\hline$N$ & \multicolumn{2}{|c|}{2932} & \multicolumn{2}{c|}{2736} & \multicolumn{2}{c|}{2697} & \multicolumn{2}{c|}{2658} \\
\hline Model & 2 -state & $\mathrm{kt}$ & 2-state & $\mathrm{kt}$ & 2-state & $\mathrm{kt}$ & 2-state & $\mathrm{kt}$ \\
\hline$n M B E$ & 0.000 & -0.006 & 0.001 & 0.005 & -0.001 & -0.016 & 0.001 & 0.005 \\
\hline$n R M S E$ & 0.003 & 0.012 & 0.258 & 0.328 & 0.227 & 0.367 & 0.201 & 0.222 \\
\hline$P(T=5 \%)$ & 1 & 0.895 & 0.251 & 0.142 & 0.278 & 0.160 & 0.732 & 0.604 \\
\hline
\end{tabular}

\section{Results and discussions}

The forecasting model was tested on the Solar Platform against data measured on a fully monitored PV module directly connected to a pure resistive load. The model performance is illustrated on basis of data recorded in four days from 2016. As Fig. 1 indicates, these days were characterized by different solar radiative regimes, ranging from a stable one (day 09/21) to a high unstable one (day 09/17).

\subsection{Nowcasting solar irradiance}

Based on the results from [6], the ARIMA $(0,1,0)$ model was used for nowcasting $S S N$. The clear-sky solar irradiance ( $G_{0, t}$ in Eq. 5) was estimated with the Biga and Rosa's [12] empirical model. The cloud transmittance $\bar{\tau}_{c}$ was estimated as follow. As stated in Sec. 3, on the Solar Platform data are recorded $15 \mathrm{~s}$ time interval. In every day, at the moment when the sun elevation angle reaches $5^{\circ}$, the cloud transmittance is being initialized to a mean value $\bar{\tau}_{c}=0.29$. Further during the day, $\bar{\tau}_{c}$ is periodically updated. At each forecasting moment, the previous values of $S S N$ are tested to find if there were a moment with $S S N=0$. If yes, $\bar{\tau}_{c}$ is updated. If not, the last value $\bar{\tau}_{c}$ is kept. In this way, $\bar{\tau}_{c}$ is always estimated over the most recent measurements and the most suitable value is provided in Eq. (5). A similar procedure was applied to estimate $c_{c s}$, but using $c_{c s}=1$ as seed in the early morning.

In a second stage of the test, the performance of the 2 -state model v. 2 was compared to the one of a standard solar irradiance forecasting model. The ARIMA model applied to the clearness index series is considered as one of the main competitors. The statistical software Statgraphics was employed for fitting the ARIMA models on $k t$ data series According to AIC criterion, the model selected for forecasting $k t$ was $\operatorname{ARIMA}(2,1,2)$. The performances of the two-state v.2 model and $k t$-based model are compared in Table 1. Considering any statistical indicator the two-state model performs more accurate than the $k \mathrm{t}$-based model. 


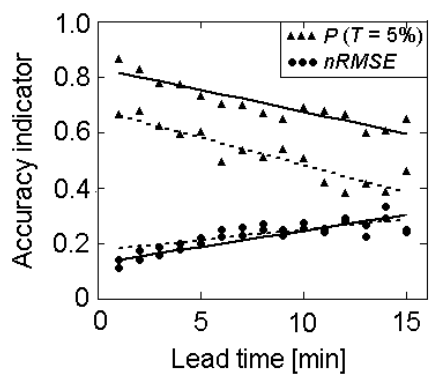

Fig. 3. Accuracy of nowcasting solar irradiance by the two-state model (line) and $k t$-based model (dashed) in 09/23 with respect to the lead time.

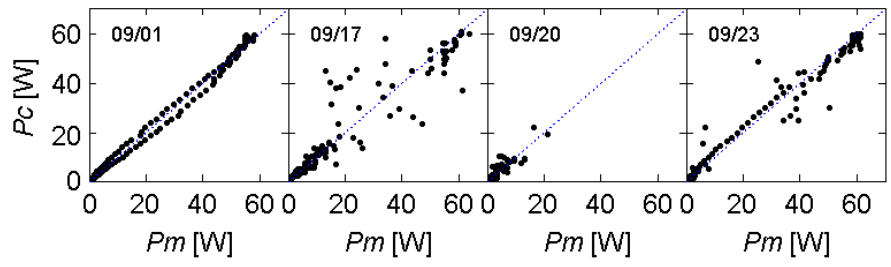

Fig. 4. Accuracy of nowcasting the output power of the monitored PV system. Lead time $=5$ minutes.

If the percentage of forecasts accurate to within a given interval is assessed, a remarkable advantage of the two-state model is noticed. This is more visible in Fig. 2, where the percentage $P$ of forecasts accurate to a given interval tolerance interval $T$ is plotted against $T$, expressed in percent. In general, for $\mathrm{T}<20 \%$, the percentage of forecasts accurate to within $T$ is far greater in the case of the two-state model. At $T=40 \%$ the success rates of the two models become comparable, but the two-state model still preserves an advance. It is worth to note that the two-state model performs accurate even in a very unstable solar radiative regime (Fig. 2, day 09/17).

Figure 3 shows the models performance in respect to the forecasting lead time in 09/23, a day with a stable solar radiative regime in the morning and high variable one afternoon. Visual inspection shows that while the two models exhibit the same accuracy (measured by $n R M S E$ ), a remarkably higher precision (measured by $P$ ) of the two-state model is noted. For the entire range of lead time considered in the illustration from Fig. 3, the two-state model precision $P(T=5 \%)$ exceeds with $20 \%$ the precision of the $k t$-based model.

\subsection{Nowcasting the PV output power}

Figure 4 assesses graphically the accuracy of the proposed procedure in nowcasting the output power of the monitored PV system for a lead time of 5 minutes. The performance is high in 09/01, a clear-sky day characterized by a stable solar radiative regime $(\overline{\operatorname{SSSN}}=0)$. It decreases with the increasing of cloudiness and the instability of the solar radiative regime. The lowest performance is noted in $09 / 17$, a day characterized by a high unstable solar radiative regime $(\overline{S S S N}=0.018)$. This behavior is induced by the decreasing of the ARIMA model accuracy in nowcasting $S S N$ with the increasing of the instability of the solar radiative regime. The cause is the model inertia, tending to preserve the current state of $S S N$ [13]. Although many efforts were devoted for improving the accuracy of nowcasting sunshine number [3], further research for removing the persistence are required. 


\section{Conclusions}

In this study an innovative procedure for nowcasting the output power of a PV system was reported. This is based on an upgraded version of the two-state model for nowcasting solar irradiance. Overall results show that the two-state model performs better in nowcasting solar irradiance than the main competitor, ARIMA forecasting of the clearness index. Always when sunshine number is correctly predicted, the forecasts trace with accuracy the measurements. This give a significative advantage to the two-state model when the percentage of the forecasts accurate to within a given tolerance interval is evaluated. A notable advantage of the two-state model is its modularity, which allows us to increase the forecasting accuracy by fine tuning its components: the clear-sky model, the dynamic adjustment of the atmospheric transmittance and the model for nowcasting sunshine number. The proposed procedure provides high-quality forecasts of the PV output power when high and moderate values of solar irradiance are recorded. Further research for improving the forecasting accuracy in low irradiance conditions and/or high unstable solar radiative regime are required.

\section{Acknowledgements}

This work was supported by a grant of the Romanian National Authority for Scientific Research and Innovation, project number PN-III-P2-2.1-PED-2016-0592.

\section{References}

1. A. Mills, M. Ahlstrom, M. Brower, A. Ellis, R. George, T. Hoff, B. Kroposki, C. Lenox, N. Miller, M. Milligan, J. Stein, Y. Wan. IEEE Power Energy M. 9, 33 (2011).

2. M. Brabec, M. Paulescu, V. Badescu. Sol Energy 101, 272 (2014).

3. Y. Chu, H.T.C.Pedro, M. Li, C.F.M.Coimbra. Sol Energy 114, 91 (2015).

4. O. Mares, M. Paulescu, V. Badescu V. Energ Convers Manage 105, 139 (2015).

5. M. Paulescu, V. Badescu, C. Dughir. Energy 70, 49 (2014).

6. M. Paulescu M, O. Mares, E. Paulescu, N. Stefu, A. Pacurar, D. Calinoiu, P. Gravila, N. Pop, R. Boata. Energ Convers Manage 79, 690 (2014).

7. V. Badescu, M. Paulescu. Atmos Res 101, 194 (2011).

8. World Meteorological Organization. Guide to Meteorological Instruments and Methods of Observation. WMO-No.8/2008.

9. M. Paulescu, V. Badescu. Theor Appl Climatol 103, 459 (2011).

10. B.Y. Liu, R.C. Jordan. Sol Energy 4, 1 (1960).

11. D. Costa, O. Mares. AIP Conference Proceedings 1634, 151 (2014).

12. A.J. Biga, R. Rosa. Sol. Energy 23, 61 (1979).

13. M. Paulescu, V. Badescu, M. Brabec. Energy 54, 104 (2013). 